\title{
Performance Evaluation of IEEE 802.15.6 Under Non-Saturation Condition
}

\author{
Saeed Rashwand \\ Department of Computer Science \\ University of Manitoba
}

\author{
Jelena Mišić \\ Department of Computer Science \\ Ryerson University
}

\begin{abstract}
A wireless body area network (WBAN) aims to provide real-time and ubiquitous body monitoring to perform autonomous early prediction, diagnostic, and tracking the response of the body to treatments. IEEE 802.15.6 is a communication standard for WBANs which pervasively and continuously monitor the human body. In this paper, we develop an analytical model for performance evaluation of the IEEE 802.15.6 standard under non-saturation regime. We validate the analytical results by a simulation model. The result of the paper indicates that adopting appropriate EAPs and RAPs lengths noticeably improves the performance of the network. The short EAPs and RAPs result in wasting the network resources due to increasing the time intervals in which the medium is inaccessible. On the other side, long EAPs and RAPs increase the collision probability at the end of EAPs and may result in undesirable frame delays for highest user priority nodes.
\end{abstract}

\section{INTRODUCTION}

Increasing in ageing population, the cost of healthcare, and the extensive benefits of a continuous and unobtrusive human well-being monitoring system have triggered the concept of the wireless body area networks (WBANs). IEEE 802.15.6 [1] is a proprietary standard for WBANs which is a short range, low power, and highly reliable wireless communication in the vicinity of, or inside, a human body. Quality of service (QoS), extremely low power and simultaneously complying with strict non-interference guidelines are the main goals of the standard.

WBANs have attracted a lot of attention during the last few years. Wireless sensors and wearable materials developmentand WBAN implementations are among the conducted researches regarding the WBANs [2], [3], [4]. The authors in [5], [6] provide extensive WBAN-related literature reviews. However, there are few works which evaluate the performance of an IEEE 802.15.6-based WBAN. In [7], [8] we studied the performance of the IEEE 802.15.6 standard under saturation condition and an error-prone channel. We showed that under saturation condition all the user priorities (UPs), except the highest UP, severely starve and the medium is very poorly utilized.

In this work, we investigate the performance of the IEEE 802.15.6 standard under non-saturation regime. A node is saturated when its frame arrival rate exceeds or is equal to its frame departure rate. We develop an analytical model which is composed of three inter-related sub-models; Markov chain, Backoff duration, and queuing models. We consider all the eight UPs $\left(U P_{k}, k=0, \ldots, 7\right)$ and the first exclusive and random access phases (EAP1 and RAP1), as specified in the standard. Based on the models we obtain a set of equations which is solved to compute mean response time of the data frames. To our best knowledge, this is the first work in the literature which investigates the performance of the IEEE 802.15.6 standard under non-saturation regime.

The remainder of this paper is organized as follows: Section II briefly introduces the IEEE 802.15.6 standard. In Section III the analytical model is developed. In Section IV we analyze the performance of an IEEE 802.15.6-based WBAN through the mean response time of data frames for all UPs. Finally, Section V concludes the paper and highlights some avenues for future research.

\section{IEEE 802.15.6 STANDARD}

In this section, we briefly describe the IEEE 802.15.6 standard. A more extensive description of the standard can be found in [7]. A WBAN is composed of logical sets called body area networks (BANs) including a single hub as the coordinator and up to 64 nodes. Eight user priorities are defined in the standard; Background $\left(U P_{0}\right)$, Best effort $\left(U P_{1}\right)$, Excellent effort $\left(U P_{2}\right)$, Controlled load $\left(U P_{3}\right)$, Video $\left(U P_{4}\right)$, Voice $\left(U P_{5}\right)$, Media data or network control $\left(U P_{6}\right)$, and Emergency or medical event report $\left(U P_{7}\right)$. They are differentiated based on the minimum and maximum contention windows, $\left(C W_{\min }, C W_{\max }\right)$; $(16,64),(16,32),(8,32),(8,16)$, $(4,16),(4,8),(2,8)$, and $(1,4)$, respectively.

The time axis is divided into beacon periods (superframes) by the hub regardless of whether it is to transmit beacon to provide or support time reference allocations in its BAN. A superframe includes Exclusive Access Phases (EAP1 and EAP2), Random Access Phases (RAP1 and RAP2), type-I/II Access Phases, and Contention Access Phase (CAP) - in the order shown in Fig. 1. Except RAP1 all other phases may have a zero length. In this work, we set the lengths of all the access phases, except EAP1 and RAP1, to 0 . We consider the CSMA/CA MAC mechanism running in narrowband PHY, as described in the standard.

EAPs can be only accessed for transmitting the $U P_{7}$ frames while all UPs are allowed to access the medium during RAPs.

At the beginning of every backoff phase a random number is uniformly drawn from the contention window (CW), $\left[1, C W_{k, i}\right]$, as the backoff count for a node of $U P_{k}$, where $C W_{k, i}=W_{k, i}$ for $i=0 . . R$. The data frame is dropped if the 


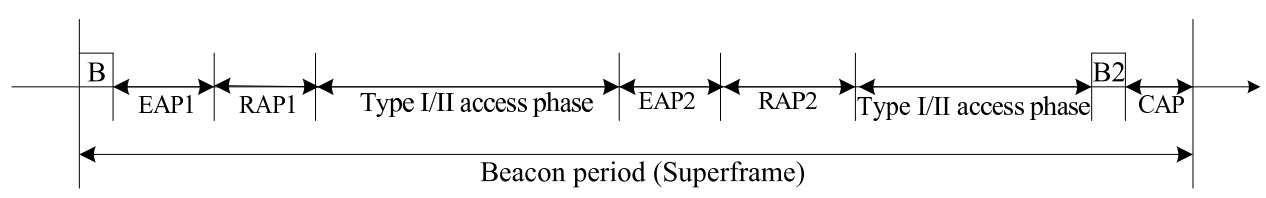

Fig. 1. Layout of access phases in a beacon period (superframe) for beacon mode

number of retransmissions during a backoff process exceeds the maximum retry limit $R$. The contention window for a node of $U P_{k}$ during the $i$-th backoff phase is obtained as follows:

- $W_{k, 0}=W_{k, \min }=C W_{k, \min }$.

- $W_{k, i}=\min \left\{2 W_{k, i-1}, C W_{k, \max }\right\}$ for $2 \leq i \leq R$ if $i$ is an even number.

- $W_{k, i}=W_{k, i-1}$ for $1 \leq i \leq R$ if $i$ is an odd number.

where $C W_{k, \min }=W_{k, 0}$ and $C W_{k, \max }=W_{k, m_{k}}$ indicate the maximum and minimum contention window sizes for a node of $U P_{k}$.

The node must lock its backoff counter when any of the following events occurs:

- The backoff counter is reset upon decrementing to 0 .

- The channel is busy due to a transmission on the medium.

- The current time is outside the access phases where the node can transmit. Any RAP or CAP if UP does not have the highest value, or is outside any EAP, RAP, or CAP if UP has the highest value.

- The current time is at the start of a CSMA slot within an EAP, RAP, or CAP, but the time between the end of the slot and the end of the EAP, RAP, or CAP is not long enough to complete a frame transaction.

The node unlocks its backoff counter when both of the following conditions are met:

- The channel has been idle for SIFS within an access phase in which the node can access the medium.

- The time duration between the current time plus a CSMA slot and the end of the EAP, RAP, or CAP is long enough to complete a frame transaction.

Upon unlocking the backoff counter, the node decreases its backoff counter by one for each idle CSMA slot that follows.

\section{Analytical Model}

In this section, we develop an analytical model to evaluate performance of the IEEE 802.15.6 standard under nonsaturation regime while the channel condition is ideal. The network is assumed to be single hop including a hub as the coordinator and $n_{k}, k=0 . .7$, nodes of $U P_{k}$ which operates in $2.4 \mathrm{GHz}$ ISM band. The CSMA slots have then the length of $\omega=125 \mu \mathrm{sec}$. We define $\lambda_{k}, k=0 . .7$, as the probability of a data frame arrival during a CSMA slot for a node of $U P_{k}$. It is clear that the inter-arrival time $I_{k}$ is geometrically distributed with a mean of $\frac{1}{\lambda_{k}}$ slots. The lengths of EAP1 and RAP1 are indicated by eap and rap in slots, respectively.

All the nodes in the model deploy RTS/CTS mechanism to access the medium. The sizes of RTS and CTS are represented by $r t s$ and $c t s$ in slots, respectively. We assume that the control frames and headers are transmitted at $91.4 \mathrm{kbps}$ while the payload of the data frames is transmitted at $971.6 \mathrm{kbps}$. The size of the data frames for a node of $U P_{k}$ is denoted by $l_{k}$ in slots and ack indicates the size of an ACK in slots.

The analytical model consists of three inter-related submodels; Markov chain, backoff duration, and queuing models; In Markov chain model, we develop eight dependent three dimensional discrete time Markov chains (DTMCs) to compute the access probabilities of all UPs following the backoff procedure of the CSMA/CA mechanism of the standard. In backoff duration model, we extend the DTMCs to fourdimensional DTMCs to calculate the backoff durations of all UPs. In queuing model, we formulate the probability that the queue is empty after a service completion time.

Based on the model, we acquire a set of equations which is analytically solved to compute the unknown variables. To be able to analytically solve the set of equations we use an iterative approach which will be discussed in detail as we go through the model.

\section{A. Markov Chain Model}

In the Markov chain model we calculate the medium access probabilities of the nodes with $U P_{k}, \tau_{k}, k=0 . .7$, by solving a set of eight dependent DTMCs. $\tau_{k}$ denotes the probability that the medium is accessed by a node of $U P_{k}$ given that the medium is not set to busy due to either a transmission on the medium or the CSMA slot is outside the access phases in which the node is allowed to transmit. The DTMC for a node of $U P_{k}$ is depicted in Fig. 2. The intervals between two successive Markov points in a DTMC may have different lengths. The Markov chain represents a random process with stationary distribution $b_{k, i, j}$, where $k=0 . .7$ indicates the user priority that the node belongs to, $i=0 . . m_{k} . . R$ denotes the backoff phase in the backoff procedure invoked by the node, and $j=0 . . W_{k, i}$ indicates the value of the backoff counter.

Probability that the medium remains idle during a slot in the RAP1, where all the UPs are allowed to transmit, is computed as follows:

$$
f=\prod_{i=0}^{7}\left(1-\tau_{i}\right)^{n_{i}}
$$

During the backoff countdown of a node of $U P_{k}$ the probability that the medium remains idle is given by

$$
f_{k}=\frac{\prod_{i=0}^{7}\left(1-\tau_{i}\right)^{n_{i}}}{\left(1-\tau_{k}\right)}, \quad k=0 . .7
$$




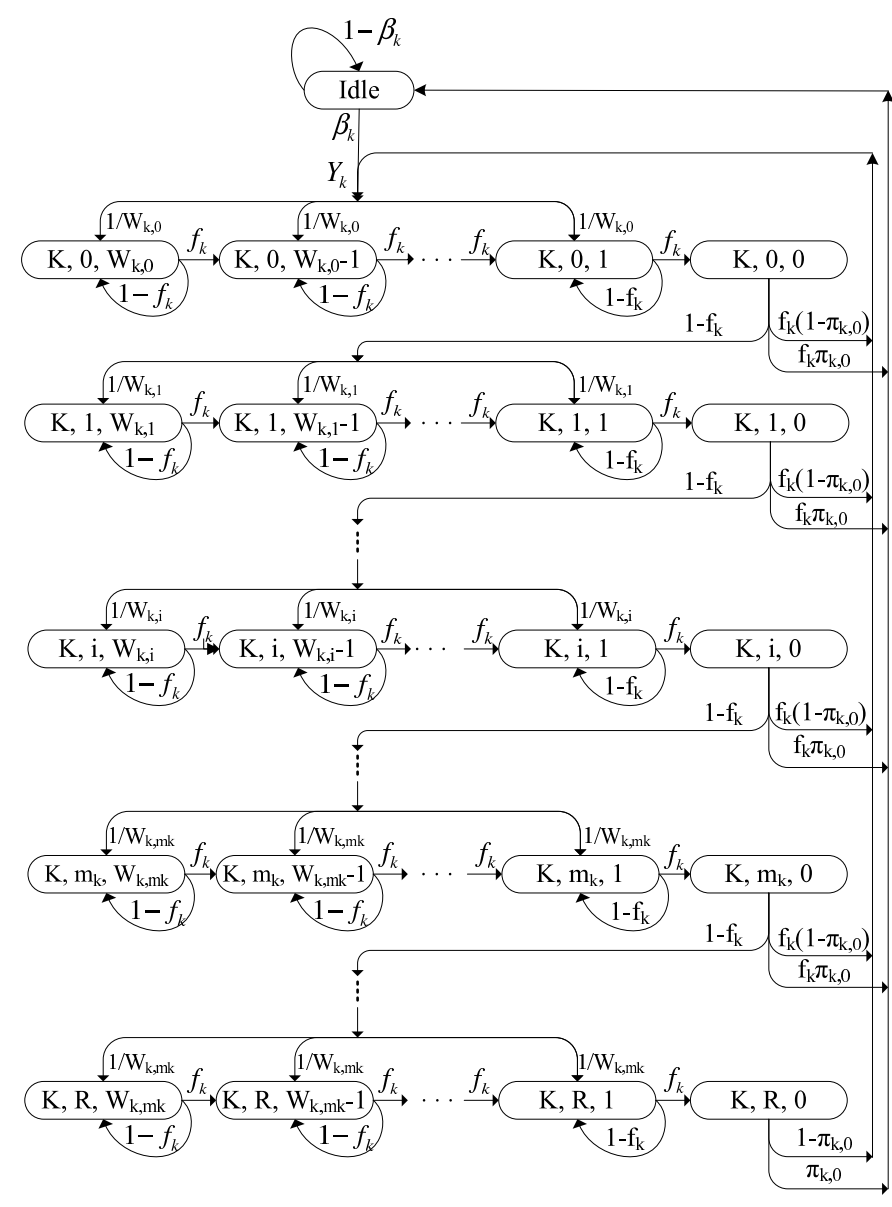

Fig. 2. Markov chain for $U P_{k}$

Because only the nodes of the highest UP are allowed to transmit on the medium during EAP1 while all UPs can access the medium during RAP1, the probability that the medium remains idle in a CSMA slot during EAP1 and RAP1 are different. We approximate the probability for a node of $U P_{7}$ during its backoff countdown as follows:

$$
f_{7}=\frac{X_{R} \prod_{i=0}^{7}\left(1-\tau_{i}\right)^{n_{i}}}{\left(X_{E}+X_{R}\right)\left(1-\tau_{7}\right)}+\frac{X_{E}\left(1-\tau_{7}\right)^{n_{7}-1}}{X_{E}+X_{R}}
$$

where $X_{E}$ and $X_{R}$ are the mean numbers of CSMA slots in EAP1 and RAP1, respectively, which are the Markov points. In our iterative approach we set the initial values of the numbers to $X_{E}=e a p$ and $X_{R}=r a p$. The values of $X_{E}$ and $X_{R}$ for the next iterations are calculated as following

$$
X_{E}=\frac{\frac{e a p}{\chi+n_{7} \tau_{7} \psi \delta L_{s}+\left(1-\chi-n_{7} \tau_{7} \psi \delta\right) L_{c}}}{f+\sum_{t=0}^{7} n_{t} \tau_{t} f_{t} \delta L_{s}+\left(1-f-\sum_{t=0}^{7} n_{t} \tau_{t} f_{t} \delta\right) L_{c}}
$$

where $\chi=\left(1-\tau_{7}\right)^{n_{7}}, \psi=\left(1-\tau_{7}\right)^{n_{7}-1}, L_{s}=$ rts + cts + $l_{k, o}+a c k+3$ sifs indicates the successful transmission time in slots, $L_{c}=r t s+c t s+$ sif $s$ is the unsuccessful transmission time in slots in case of failure access to the medium. We define $l_{k, o}, k=0 . .7$ as the average size (in slots) of a data frame transmitted by other nodes in the network because UPs may have different data frame sizes. The subscript 'o' indicates the word "others". The initial values for $l_{k, o}$ are defined as follows:

$$
l_{k, o}=\frac{\sum_{u=0}^{7} n_{u} l_{u}-l_{k}}{\sum_{u=0}^{7} n_{u}-1}, \quad k=0 . .7
$$

We update the values of $l_{k, o}$ in the next iteration as follows:

$$
l_{k, o}=\frac{\sum_{u=0}^{7} n_{u} \tau_{u} l_{u}-\tau_{k} l_{k}}{\sum_{u=0}^{7} n_{u} \tau_{u}-\tau_{k}}, \quad k=0 . .7
$$

We introduce $p_{k}$ to address the probability that in a given CSMA slot the node notices that there is not enough time during the current access period to complete a frame transaction. Hereafter, the backoff counter remains locked until the moment when the node is allowed to transmit (RAP1 for $U P_{k}, k=0, \ldots, 6$ or EAP1 for $\left.U P_{7}\right) . p_{k}$ is computed as: $p_{k}=$ $\frac{3}{2\left(\mathrm{rap}-L_{s}-C_{k}\right)}, \quad k=0, \ldots, 6$ and $p_{7}=\frac{3}{2\left(\mathrm{rap}+\mathrm{eap}-L_{s}-C_{7}\right)}$, where $C_{k}=\frac{C W_{k, \min }+C W_{k, \max }}{4}, k=0, \ldots, 7$ approximates the mean backoff value. .

The medium access probability of a node with $U P_{k}$ is calculated as: $\tau_{k}=\sum_{i=0}^{R} b_{k, i, 0}, k=0 . .7$.

By solving the Markov chain we can show that the following equation is correct for $k=0 . .7, i=0 . . R$, and $j=1 . . W_{k, i}$.

$$
b_{k, i, j}=\frac{\left(1-f_{k}\right)^{i} Y_{k}\left(W_{k, i}-j+1\right)}{W_{k, i} f_{k}}
$$

For $k=0 . .7$ and $i=0 . . R$ we have $b_{k, i, 0}=\left(1-f_{k}\right)^{i} Y_{k}$.

We define $Y_{k}, k=0 . .7$, as the input probability to the zero-th backoff phase which is computed as follows:

$$
Y_{k}=\frac{\tau_{k} f_{k}\left(1-\pi_{k, 0}\right)+p_{k, \text { Idle }} \beta_{k}}{1-\left(1-f_{k}\right)^{R+1}\left(1-\pi_{k, 0}\right)}, \quad k=0 . .7
$$

where $\pi_{k, 0}$ indicates the probability that the queue of the node with $U P_{k}$ is empty when a data frame is either successfully transmitted or dropped due to a retry limit exceed, and $p_{k, \text { Idle }}$ represents the probability of being in state "Idle" waiting for a data frame to arrive. $\pi_{k, 0}$ will be formulated in the queuing model while $p_{k, \text { Idle }}$ is calculated as follows:

$$
p_{k, \text { Idle }}=\frac{\tau_{k} f_{k} \pi_{k, 0}}{\beta_{k}\left(1-\left(1-f_{k}\right)^{R+1}\right)}, \quad k=0 . .7
$$

where $\beta_{k}$ is given by $\beta_{k}=f_{k} p_{k}(1-$ $\left.\left(1-\lambda_{k}\right)^{L_{k}}\right)+f_{k}\left(1-p_{k}\right) \lambda_{k}+(1-$ $\left.f_{k}\right)\left(p_{k} \sum_{u=0}^{L_{s}-1}\left(1-p_{k}\right)^{u}\left(1-\left(1-\lambda_{k}\right)^{L_{k}+u+1}\right)+(1-\right.$ $\left.\left.p_{k}\right)^{L_{s}}\left(1-\left(1-\lambda_{k}\right)^{L_{s}}\right)\right)$

We define $L_{k}=e a p+r t s+c t s+3$ sifs $+l_{k}+a c k, k=0 . .6$, as the number of slots in which the backoff counter of a $U P_{k}$ node must be kept locked because there is not enough time 
to complete a data frame transaction. A $U P_{7}$ node does not require to lock its backoff counter during EAP1 unless the medium is busy, resulting in $L_{7}=r t s+c t s+3 s i f s+l_{k}+a c k$. In case of having the backoff counter equal to $j$, a node has to keep it's counter locked for $L_{k}+j$ slots if the node notices that there is not enough time to complete a data frame transaction.

Based on the normalization condition of DTMCs summation of all the probabilities is equal to 1 which results in the following set of eight equations for $k=0 . .7$ :

$$
1=p_{k, I d l e}+Y_{k} \sum_{i=0}^{R}\left(1-f_{k}\right)^{i}\left(1+\sum_{j=1}^{W_{k, i}} \frac{W_{k, i}-j+1}{W_{k, i} f_{k}}\right)
$$

\section{B. Backoff Duration Model}

In order to accurately compute the probability that the queue is empty after a successful data frame transmission or a data frame drop, we must calculate the backoff duration of all UPs. In this section, we calculate the average durations of every backoff phase and the total backoff period before successfully accessing the medium or dropping the data frame using probability generating functions (PGFs).

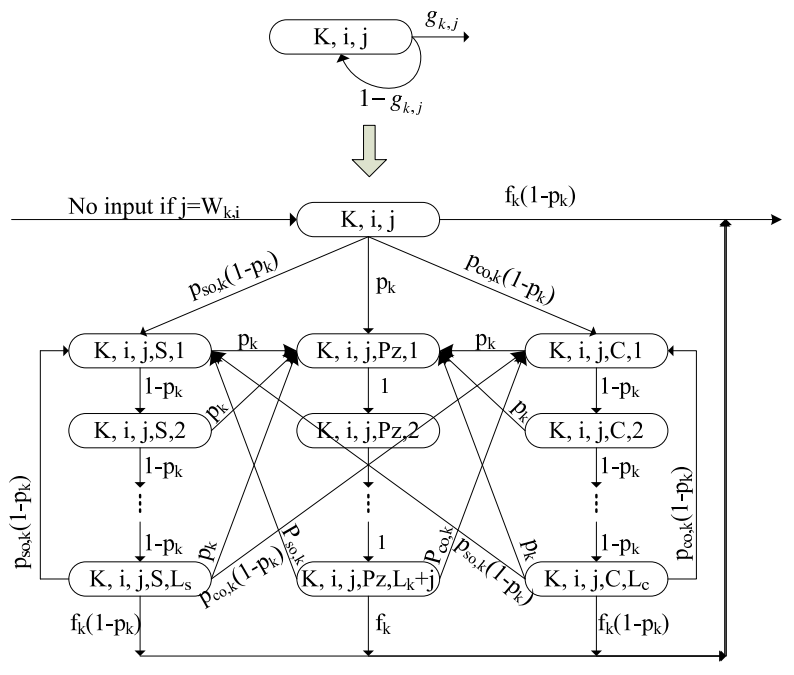

Fig. 3. Markov chain extension for $U P_{k}$

To calculate the length of backoff durations we require to extend the DTMCs, described in section III-A, to ones that beginning of every CSMA slot represents a Markov point. In the extended DTMCs the distance between every two Markov points is a slot. To compose the extended four dimensional DTMCs we replace the component shown on top of Fig. 3 with the component shown below that in the three dimensional DTMCs (Fig. 2) for $k=0 . .7, i=0 . . R$, and $j=0 . . W_{k, i}$.

Stationary distributions of $b_{k, i, j, S, t}, t=1 . . L_{s}$, and $b_{k, i, j, C, t}, t=1 . . L_{c}$, correspond to the slots during the time period in which the data frame is successfully transmitted and the time period in which the RTS frame collides, respectively. Stationary distribution of $b_{k, i, j, P z, t}$ for $t=1 . . L_{k}+j$ corresponds to a slot during the time period in which the node's backoff counter is locked because there is not enough time to complete a data frame transaction.

We refer $p_{s o, k}, k=0 . .7$, and $p_{s o, k}, k=0 . .7$, as the probabilities that the medium becomes busy due to a successful transmission by another node and unsuccessful accesses by other nodes in the network, respectively, which are given by $p_{s o, k}=\sum_{i=0}^{7} \frac{n_{i} \tau_{i} f_{k}}{1-\tau_{i}}-\frac{\tau_{k} f_{k}}{1-\tau_{k}}$ and $p_{c o, k}=1-f_{k}-p_{s o, k}$.

We indicate the PGFs for durations of a data frame transaction and a failed transmission due to an RTS collision by $S t_{k}(z)=z^{r t s+c t s+l_{k}+a c k+3 s i f s}$ and $C t(z)=z^{r t s+c t s+s i f s}$, respectively. We define $B f S_{k, j}(z)$ and $B f C_{k, j}(z)$ as the PGFs of the time durations from the moment when the backoff counter is locked due to a successful transmission by another node or an unsuccessful medium access by the other nodes, respectively, until the moment when the backoff counter is unlocked. We introduce $B f p z_{k, j}(z)$ as the PGF of the time duration from the moment when the backoff counter is locked because there is not enough time to complete a frame transaction until the moment when the backoff counter is unlocked. The PGFs are computed using the extended DTMCs as follows:

$$
\begin{aligned}
B f p z_{k, j}(z)= & z^{L_{k}+j}\left(f_{k} z+\Theta_{k, j}(z)\right) \\
\operatorname{BfS} S_{k, j}(z)= & p_{k} B f p z_{k, j}(z) \frac{1-\left(1-p_{k}\right)^{L_{s}} z^{L_{s}}}{1-\left(1-p_{k}\right) z}+ \\
& \left(1-p_{k}\right)^{L_{s}} f_{k} z^{L_{s}}+\left(1-p_{k}\right)^{L_{s}} \Theta_{k, j}(z) \\
B f C_{k, j}(z)= & p_{k} B f p z_{k, j}(z) \frac{1-\left(1-p_{k}\right)^{L_{c}} z^{L_{c}}}{1-\left(1-p_{k}\right) z}+ \\
& \left(1-p_{k}\right)^{L_{c}} f_{k} z^{L_{c}}+\left(1-p_{k}\right)^{L_{c}} \Theta_{k, j}(z)
\end{aligned}
$$

where $\Theta_{k, j}(z)=p_{s o, k} B f S_{k, j}(z)+p_{c o, k} B f C_{k, j}(z)$. $B f S_{k, j}(z), B f C_{k, j}(z)$, and $B f p z_{k, j}(z)$ can be analytically written based on the known parameters. We do not present the formulae here because they are large and the reader can simply derive them. The PGF of the time to decrease the backoff counter by one, where the counter is equal to $j$, is given by denoted by $\Phi_{k, i, j}=p_{k} B f p z_{k, j}+\left(1-p_{k}\right)\left(f_{k} z+\Theta_{k, j}(z)\right)$ for $k=0 . .7, j=1 . . W_{k, i}, i=0 . . R$. we are now able to write the PGF of the $i$-th backoff phase duration as follows:

$$
\begin{aligned}
\Phi_{k, i} & =\sum_{j=1}^{W_{k, i}} \prod_{t=1}^{j} \Phi_{k, i, t}\left(f_{k}\left(L_{s} p_{k} z^{L_{k}}+1-L_{s} p_{k}\right)\right. \\
& \left.+\left(1-f_{k}\right)\left(L_{c} p_{k} z^{L_{k}}+1-L_{c} p_{k}\right)\right) / W_{k, i}
\end{aligned}
$$

The backoff process duration PGF for a $U P_{k}$ node equals:

$$
\begin{aligned}
& \Phi_{k}(z)=\sum_{i=0}^{m_{k}}\left(\prod_{u=0}^{i} \Phi_{k, u}\right)\left(1-f_{k}\right)^{i}\left(z^{L_{c}}\right)^{i} f_{k}+ \\
& \sum_{\substack{i=m_{k}+1 \\
m_{k}}}^{R}\left(\prod_{u=0}^{m_{k}} \Phi_{k, u}\right) \Phi_{k, m_{k}}^{i-m_{k}}\left(1-f_{k}\right)^{i}\left(z^{L_{c}}\right)^{i} f_{k}+ \\
& \left(\prod_{u=0}^{\prod_{k, u}} \Phi_{k, m_{k}} \Phi_{k-m_{k}}^{R}\left(1-f_{k}\right)^{R+1}\left(z^{L_{c}}\right)^{R+1}\right.
\end{aligned}
$$


Using the equation (13) we calculate the mean backoff time before the medium is successfully accessed or the data frame is dropped for a $U P_{k}, k=0 . .7$, as follows:

$$
\phi_{k}=\left.\frac{d}{d z} \Phi_{k}(z)\right|_{z=1}
$$

\section{Queuing Model}

For every node in the WBAN we model the queue of the node using a pure limited $\mathrm{Geo} / \mathrm{G} / 1$ system with vacation [9]. The Geo/G/1 queue is a single-server discrete time system with a Bernoulli arrival process and generally distributed service times. In our analytical model we assume infinite capacity for every queue. We assume that upon successful accessing to the medium every node transmits a single data frame. We denote by $B_{k}(z)=\Phi_{k}(z) S t_{k}(z), k=0 . .7$, as the probability distribution of service time (in slots) of a data frame for a $U P_{k}$ node, which includes a backoff process and successful transmission time. Hence, we have The mean of the service time distribution for a node of $U P_{k}$ is given by $b_{k}=\phi_{k}+s t_{k}$, where $s t_{k}=r t s+c t s+l_{k}+a c k+3 s i f s$. The offered load or traffic intensity of the queue, $\rho_{k}$, is assumed to be less than unity for stability condition:

$$
\rho_{k}=\lambda_{k} b_{k}<1
$$

Using the approach described in [9] we calculate the probability that the queue would be empty after a successful data frame transmission or a data frame drop as follows:

$$
\pi_{k, 0}=\left(1-\rho_{k}\right)\left\{1-I f_{k}\left(1-\lambda_{k}\right)\right\}, \quad k=0 . .7
$$

where $I f_{k}(z)=\frac{\lambda_{k} z}{1-\left(1-\lambda_{k}\right) z}$. From (10) and (16) we obtain 16 equations which enable us to calculate the 16 unknown variables of $\tau_{k}$ and $\pi_{k, 0}$ for $k=0 . .7$. As previously mentioned to be able to analytically solve the model we employ an iterative approach. We first solve the model based on the initial values for $X_{E}, X_{R}$, and $l_{k, o}, k=0 . .7$. The next iterations are performed using the computed values for unknown variables.

By calculating the unknown variables in the analytical model, we are able to compute the mean response time of a $U P_{k}$ data frame as follows [9]:

$$
\zeta_{k}=\rho_{k}+\frac{\lambda_{k}^{2} b_{k}^{(2)}-\lambda_{k} b_{k}}{2\left(1-\rho_{k}\right)}+\frac{\lambda_{k}}{2}
$$

where $b_{k}^{(2)}=b_{k}+\left.\frac{d^{2}}{d z^{2}} B_{k}(z)\right|_{z=1}$. Response time of a data frame is duration of the time interval from the moment the frame arrives to the queue to the moment when it is successfully transmitted or dropped.

\section{Performance Evaluation}

In this section, we study the performance of an IEEE 802.15.6-based WBAN under non-saturation regime. We investigate accuracy of the developed analytical model. We used Maple 13 [10] for solving the analytical model to compute the mean response time of data frames for all UPs. We
TABLE I

HEALTHCARE NODES ARE SPREAD INTO 8 UPS (NN: NUMBER OF NODES, TL: TRAFFIC LOAD PER PACKET, PS: PAYLOAD SIZE, FR: FREQUENCY RATE, AND SS: SAMPLE SIZE)

\begin{tabular}{|c|c|c|c|c|c|c|}
\hline UP & Node & NN & TL & PS & FR & SS \\
\hline 7 & ECG & 1 & $2 \mathrm{p} / \mathrm{s}$ & $150 \mathrm{~B}$ & $200 \mathrm{~Hz}$ & $12 \mathrm{~b}$ \\
& EEG & 1 & $2 \mathrm{p} / \mathrm{s}$ & $150 \mathrm{~B}$ & $200 \mathrm{~Hz}$ & $12 \mathrm{~b}$ \\
\hline 6 & ECG & 2 & $2 \mathrm{p} / \mathrm{s}$ & $150 \mathrm{~B}$ & $200 \mathrm{~Hz}$ & $12 \mathrm{~b}$ \\
& Blood Pressure & 1 & $2 \mathrm{p} / \mathrm{s}$ & $150 \mathrm{~B}$ & $200 \mathrm{~Hz}$ & $12 \mathrm{~b}$ \\
\hline 5 & EEG & 3 & $2 \mathrm{p} / \mathrm{s}$ & $150 \mathrm{~B}$ & $200 \mathrm{~Hz}$ & $12 \mathrm{~b}$ \\
\hline 4 & Glucose & 1 & $1 \mathrm{p} / \mathrm{s}$ & $50 \mathrm{~B}$ & $50 \mathrm{~Hz}$ & $16 \mathrm{~b}$ \\
& Oxygen Saturation & 1 & $1 \mathrm{p} / \mathrm{s}$ & $50 \mathrm{~B}$ & $50 \mathrm{~Hz}$ & $16 \mathrm{~b}$ \\
& Physical Activity & 4 & $1 \mathrm{p} / \mathrm{s}$ & $50 \mathrm{~B}$ & $50 \mathrm{~Hz}$ & $16 \mathrm{~b}$ \\
\hline 3 & EMG & 2 & $4 \mathrm{p} / \mathrm{s}$ & $500 \mathrm{~B}$ & $1 \mathrm{KHz}$ & $16 \mathrm{~b}$ \\
\hline 2 & Temperature & 1 & $0.25 \mathrm{p} / \mathrm{s}$ & $20 \mathrm{~B}$ & $5 \mathrm{~Hz}$ & $8 \mathrm{~b}$ \\
& Respiration Rate & 1 & $0.25 \mathrm{p} / \mathrm{s}$ & $20 \mathrm{~B}$ & $5 \mathrm{~Hz}$ & $8 \mathrm{~b}$ \\
\hline 1 & ECG & 5 & $0.5 \mathrm{p} / \mathrm{s}$ & $600 \mathrm{~B}$ & $200 \mathrm{~Hz}$ & $12 \mathrm{~b}$ \\
\hline 0 & EEG & 8 & $0.5 \mathrm{p} / \mathrm{s}$ & $600 \mathrm{~B}$ & $200 \mathrm{~Hz}$ & $12 \mathrm{~b}$ \\
\hline
\end{tabular}

simulated the IEEE 802.15.6 standard using Opnet simulator [11] to validate the analytical results. We set the exogenous parameters according to the standard. The retry limit is set to $R=7$ for all the UPs.

The WBAN consists of a hub and 31 nodes including 12 channels for Electroencephalogram (EEG), 8 channels for Electrocardiogram (ECG), 1 blood pressure sensor, 1 glucose monitoring node, 1 blood oxygen saturation monitoring sensor (pulse oximeter), 4 physical activity monitoring sensors, 2 channels for Electromyogram (EMG), 1 body temperature sensor, and 1 respiration rate monitoring node. We spread the nodes into 8 UPs as shown in Table I [12]. In all plots in this section, the lines with the line-styles thin solid (black), dot (red), dash (blue), dash-dot (green), long-dash (gold), spacedot (khaki), space-dash (magenta), and thick solid (coral) represent user priorities, $0,1,2,3,4,5,6$, and 7, respectively.

The performance of the network is investigated in two cases. In the first case, the length of RAP1 varies from $0.1 \mathrm{sec}$ to 0.5 sec while the length of EAP1 is set to $0.05 \mathrm{sec}$. The simulation and analytical results of the first case are shown in Fig. 4. The results indicate that increasing the length of RAP1 where the EAP1 length is constant improves the frame delay for all UPs. The reason is that having longer RAP periods compared to EAP periods decreases the collision probability after an EAP period. It also shows that having short EAP-RAP periods increases the mean response time of the data frames.

In the second case, the length of EAP1 varies from 0.025 $\mathrm{sec}$ to $0.20 \mathrm{sec}$ while the length of RAP1 is set to $0.2 \mathrm{sec}$. The simulation and analytical results are depicted in Fig. 5. The results show how the mean response time of all the UPs data frames increases when the length of EAP1 increases. The main reason is that because the network is unsaturated having a long EAP period wastes the network resources during the exclusive access phases. However, during RAPs the traffic congestion increases because other nodes have shorter time periods for transmission. Therefore, adopting appropriate RAP and EAP periods noticeably improves the performance of an IEEE 802.15.6-based WBAN.

The results also indicate a reasonably good match between 


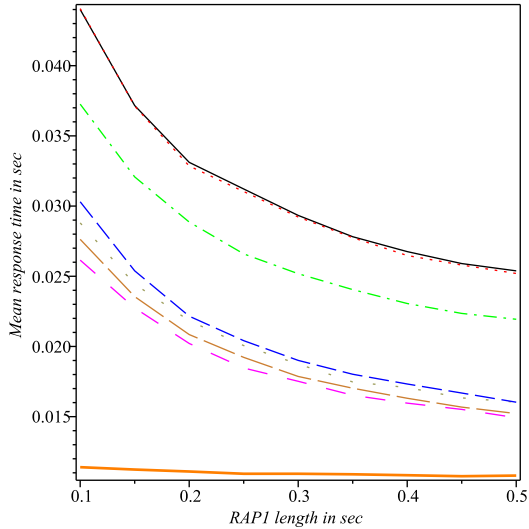

(a) Simulation Results

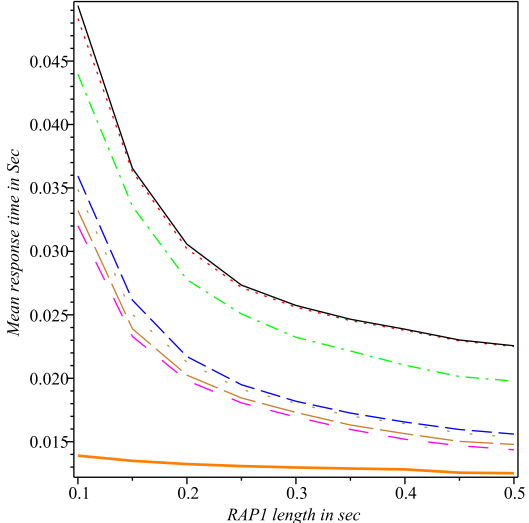

(b) Analytical Results

Fig. 4. Mean response time of data frames when length of RAP1 varies (length of EAP1 $=0.05 \mathrm{sec}$ )

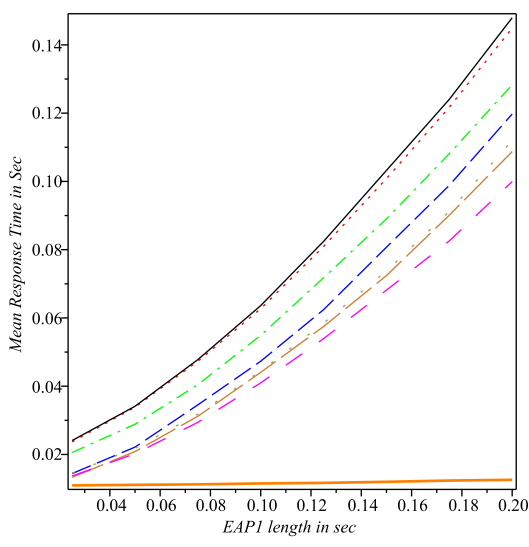

(a) Simulation Results

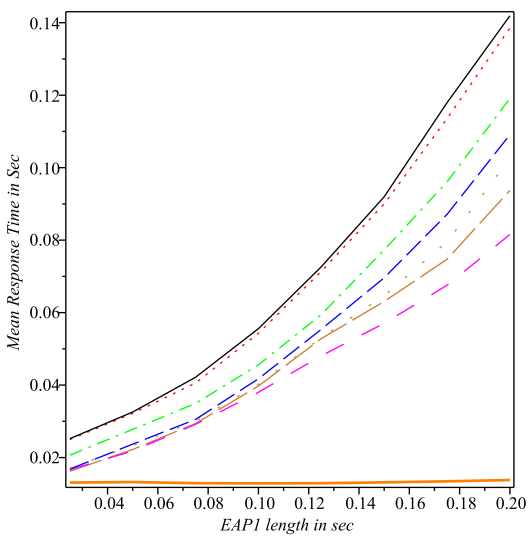

(b) Analytical Results

Fig. 5. Mean response time of data frames when length of EAP1 varies (length of RAP1 $=0.2 \mathrm{sec}$ )

the simulation and analytical results.

\section{CONClusion}

In this paper, we developed an analytical model for performance evaluation of the IEEE 802.15.6 standard under nonsaturation regime. We calculated the mean response time of the data frames in the network, which is known as the most important network performance descriptor. We validated the analytical results by an accurate simulation model. The results of this study indicate that setting the lengths of the access phases according to the traffic load of the network considerably improves the performance of the network. The models will be extended in our future work to consider other access phases and a noisy channel.

\section{REFERENCES}

[1] Wireless Body Area Network Draft Standard, IEEE Std. 802.15.6, 2010.

[2] H. Chen, W. Wu, and J. Lee, "A wban-based real-time electroencephalogram monitoring system: Design and implementation," Journal of Medical Systems, vol. 34, pp. 303-311, 2010.

[3] F. E. H. Tay, D. G. Guoa, L. Xua, M. N. Nyana, and K. L. Yap, "MEMSWear-biomonitoring system for remote vital signs monitoring," Journal of the Franklin Institute, vol. 346, pp. 531-542, Aug. 2009.
[4] D. Albu, J. Lukkien, and R. Verhoeven, "On-node processing of ecg signals," in Proceedings of the 7th IEEE conference on Consumer communications and networking conference, Las Vegas, Nevada, USA, Jan. 2010, pp. 1165-1169.

[5] B. Latre, B. Braem, I. Moerman, C. Blondia, and P. Demeester, "A survey on wireless body area networks," Wireless Networks, vol. 17, Jan. 2011.

[6] A. Pantelopoulos and N. G. Bourbakis, "A survey on wearable sensorbased systems for health monitoring and prognosis," IEEE Transactions on Systems, Man, and Cybernetics, Part C: Applications and Reviews, vol. 40, Jan. 2010.

[7] S. Rashwand, J. Misic, and H. Khazaei, "IEEE 802.15.6 under saturation: Some problems to be expected," Journal of Communications and Networks, to appear, 2011.

[8] —_, "Performance analysis of IEEE 802.15.6 under saturation condition and error-prone channel," in Proc. the IEEE Wireless Communications and Networking Conference (WCNC'11), Cancun, Mexico, Mar. 2011.

[9] H. Takagi., Queueing Analysis; A Foundation of Performance Evaluation - Volume 3: Discrete-Time Systems. NORTH HOLLAND, 1991.

[10] (2010) Maple 13, maplesoft. Waterloo, Canada. [Online]. Available: http://www.maplesoft.com

[11] (2010) Opnet modeler, opnet technologies, inc. Bethesda, MD. [Online]. Available: http://www.opnet.com/

[12] J. D. Bronzino, The Biomedical Engineering Handbook - Third Edition. Volume 2: Medical Devices and Systems. Taylor \& Francis Group, LLC, 2006. 